\title{
Prospective study of health-related quality of life after RouX-en-Y bypass surgery for morbid obesity
}

\author{
C.-Y. Chang1, ${ }^{1,3,5}$, C.-K. Huang ${ }^{2}$, Y.-Y. Chang ${ }^{5}$, C.-M. Tai ${ }^{1}$, J.-T. Lin ${ }^{1,3}$ and J.-D. Wang $^{3,4,5}$ \\ Departments of ${ }^{1}$ Internal Medicine and ${ }^{2}$ Surgery, E-Da Hospital, I-Shou University, Kaohsiung, and Departments of ${ }^{3}$ Internal Medicine and \\ ${ }^{4}$ Environmental and Occupational Medicine, National Taiwan University Hospital, and ${ }^{5}$ Institute of Occupational Medicine and Industrial Hygiene, \\ College of Public Health, National Taiwan University, Taipei, Taiwan \\ Correspondence to: Dr J.-D. Wang, Department of Internal Medicine, National Taiwan University Hospital and Institute of Occupational Medicine and \\ Industrial Hygiene, College of Public Health, National Taiwan University, Room 719, No. 17, Shiujou Road, Taipei, Taiwan (e-mail: jdwang@ntu.edu.tw)
}

\begin{abstract}
Background: The aim of this study was to evaluate the effects of Roux-en-Y gastric bypass for morbid obesity on health-related quality of life (QOL) during the first year of follow-up.

Methods: The World Health Organization Quality of Life - Brief (WHOQOL-BREF) was administered 1 month before operation, and at 1, 3, 6 and 12 months after surgery. Body mass index, co-morbidities and operation-related complications were measured at these times. A mixed-effect model was constructed to analyse repeated measurements and determine the relationships between body mass index, WHOQOLBREF scores and other variables.

Results: A total of 102 patients were enrolled. The mixed-effect model showed that the physical, psychological and social domains improved after bariatric surgery, with simultaneous reduction in weight and improvement in co-morbidities. There was a dip in scores in physical and psychological domains 3-6 months after surgery, significantly related to complications. All patients gradually improved between 6 and 12 months after surgery, reaching levels similar to those of healthy subjects.

Conclusion: Health-related QOL improved dramatically after bariatric surgery, dipped slightly between 3 and 6 months, and improved again up to the end of the first year.
\end{abstract}

Paper accepted 11 May 2010

Published online 19 July 2010 in Wiley Online Library (www.bjs.co.uk). DOI: 10.1002/bjs.7179

\section{Introduction}

Obesity has increased markedly in recent years and it is now a major public health issue in many Western countries ${ }^{1-3}$. With more Westernized dietary habits and sedentary lifestyles, obesity has also become an emerging problem in Asia $^{4}$. In Taiwan, the prevalence of obesity is 19.2 per cent for men and 13.4 per cent for women ${ }^{5}$. Many obesityrelated co-morbidities have been described and account for considerable medical resource use worldwide ${ }^{6-8}$. In general, increases in body mass index (BMI) are correlated with poorer health-related quality of life $(\mathrm{QOL})^{9-11}$.

Although non-surgical treatments for obesity can result in weight loss of approximately 5-20 per cent of bodyweight ${ }^{12,13}$, these methods are mainly ineffective in long-term resolution of excess bodyweight. Successful bariatric surgery not only helps to reduce bodyweight and co-morbidities, but it also results in an improvement in patients' health-related $\mathrm{QOL}^{9,13-19}$. Little is known, however, about changes in health-related QOL with time, probably because of difficulties in controlling for potential confounders.

The aim of this study was to evaluate the effects of bariatric surgery on changes in health-related QOL, comorbidities and loss of excess BMI during the year after operation.

\section{Methods}

This study was approved by the Institutional Ethics Committee of E-Da Hospital, I-Shou University. All patients who came to the Bariatric Centre seeking surgical treatment for morbid obesity were invited to participate. Laparoscopic Roux-en-Y gastric bypass is the standard procedure and had been performed regularly in this hospital before the start of the study. The recommendations of the Asia-Pacific consensus ${ }^{4}$ were adopted, which stipulate that to be eligible for bariatric surgery patients must be 
aged between 18 and 65 years with a BMI between 32 and $40 \mathrm{~kg} / \mathrm{m}^{2}$ and obesity-related co-morbid conditions, or a BMI above $40 \mathrm{~kg} / \mathrm{m}^{2}$. Exclusion criteria included previous gastric surgery, large hiatus hernia, history of alcohol or substance addiction, and poorly controlled non-obesityrelated medical diseases such as unresolved depression. All patients underwent psychiatric interview and those with mental illness or unrealistic expectations for surgical treatment were excluded from the study. The presence of diabetes, heart disease, hypertension, asthma, sleep apnoea, cancer and other major co-morbid conditions, as well as educational level, marital status, employment, religion, monthly income and history of smoking or drinking, were recorded. All subjects received regular follow-up for 1 year after surgery at the Bariatric Centre.

\section{Health-related quality of life questionnaire}

The World Health Organization Quality of Life - Brief (WHOQOL-BREF) ${ }^{20-23}$ is a generic QOL instrument designed to assess physical, psychological, social and environment domains. It has been shown to have good validity for use across different countries ${ }^{22}$ and different patient groups, including those with morbid obesity $^{10,24-27}$. Every subject was asked to complete the WHOQOL-BREF, Taiwan version ${ }^{28}$, in the outpatient clinic of the Bariatric Centre before the operation, and after 1, 3, 6 and 12 months. The WHOQOL-BREF is a self-completed questionnaire. It was administered by an experienced research nurse who was ready to help the subjects, should any question be raised. Subjects were encouraged to fill out every item. Questionnaires were considered effective when more than 80 per cent of items had been answered. The score of any omitted item was replaced by the average score of the other items in the same domain. The Taiwan version of the WHOQOL-BREF contains four domains (physical, psychological, social and environment), including the 26 original items of the WHOQOL-BREF, plus two Taiwanese culture-specific questions as national items. One item 'being respected and accepted' was categorized in the social domain and another addressing 'eating what one likes' was categorized in the environmental domain. The method of application, scoring procedures and reference time point (during the last 2 weeks) were the same as for the original WHOQOLBREF 23. Each item was scored from 1 to 5 points and a higher score was considered to indicate a better QOL. Because the numbers of items were different for each domain, the domain scores were calculated by multiplying the average of the scores of all items in the domain by the same factor of four. Therefore, each domain score had the same range, from 4 to 20 .

\section{Physical evaluation}

Blood pressure, complete blood count and serum biochemistry profile including aspartate aminotransferase, alanine aminotransferase (ALT), triglycerides, total cholesterol, low-density lipoprotein, high-density lipoprotein and fasting glucose were recorded before, and at 1, 3, 6 and 12 months after surgery. Bodyweight and height were measured at the same times. Abnormal liver function was defined as a serum ALT level more than two times the reference level for the E-Da Hospital. Oesophagogastroduodenoscopy was performed to detect peptic ulcer and possible infection with Helicobacter pylori, which was treated before surgery. Gastro-oesophageal reflux disease (GORD) was determined by endoscopy or the presence of typical symptoms using a specific questionnaire, the Reflux Disease Questionnaire, administered before surgery and after 1, 3, 6 and 12 months. Endoscopy was performed if upper gastrointestinal bleeding, dysphagia or epigastric discomfort developed during follow-up.

\section{Reference population}

A reference group of healthy subjects matched by age (within 5 years), sex, municipality, marriage and education was randomly selected from the database of the 2001 National Health Interview Survey (NHIS) ${ }^{29}$ conducted by the National Health Research Institutes and the Bureau of Health Promotion, Department of Health, Taiwan. The 2001 NHIS provided nationwide estimates about health conditions, health behaviours and the use of medical resources by the Taiwanese population. The WHOQOLBREF, Taiwan version, was one of the tools included in this national survey. Each morbidly obese patient was matched with two reference subjects with a BMI no greater than $32 \mathrm{~kg} / \mathrm{m}^{2}$.

\section{Statistical analysis}

A descriptive analysis was conducted comparing the demographic characteristics of patients and reference subjects; $\chi^{2}$ test was used for analysis of categorical variables and Student's $t$ test for continuous data. Different domains of WHOQOL-BREF were summarized along with repeated measurements at different times and these were then compared with values from the normal reference population in Taiwan using Student's $t$ test. Mixed-effect models were constructed assuming a linear autocorrelation for repeated measurements within individual subjects by using the summary scores for each domain and individual items as the dependent variables, whereas BMI values, time, age, sex, years of education, employment, monthly 
income, marital status, co-morbidities and operationrelated complications were included as independent predictive variables. All data were collected and analysed using SAS ${ }^{\circledR}$ software version 9.0 (SAS Institute, Cary, North Carolina, USA).

\section{Results}

All 218 consecutive patients who had surgery between January 2007 and June 2008 were invited to participate. One hundred and two patients (46.8 per cent) accepted the invitation, joined the study, and filled in the questionnaires by themselves. They underwent regular follow-up for 1 year after surgery. There were no significant differences in clinical and sociodemographic characteristics

Table 1 Demographic characteristics of patients and age-, sex-, municipality-, marriage- and education-matched healthy control subjects

\begin{tabular}{|c|c|c|c|}
\hline & $\begin{array}{l}\text { Patients } \\
\text { with obesity } \\
(n=102)\end{array}$ & $\begin{array}{l}\text { Healthy } \\
\text { subjects } \\
(n=200)\end{array}$ & $P \dagger$ \\
\hline Sex ratio (F : M) & $64: 38$ & $125: 75$ & 0.967 \\
\hline Age (years) ${ }^{\star}$ & $32 \cdot 1(9 \cdot 6)(18-54)$ & $31 \cdot 8(9 \cdot 5)(20-55)$ & $0.802 \ddagger$ \\
\hline BMI $\left(\mathrm{kg} / \mathrm{m}^{2}\right)^{*}$ & $42 \cdot 3(7 \cdot 0)(32-67)$ & $23.9(3.6)(17-32)$ & $<0.001 \neq$ \\
\hline Married & $33(32.4)$ & $64(32.0)$ & 0.951 \\
\hline Education (years) & & & 0.897 \\
\hline$\leq 12$ & $39(38.2)$ & $78(39.0)$ & \\
\hline$>12$ & $63(61.8)$ & $122(61.0)$ & \\
\hline Employed & $47(46 \cdot 1)$ & $129(64.5)$ & 0.002 \\
\hline Monthly income (NT) & & & 0.261 \\
\hline$<20000$ & $38(37.3)$ & $88(44.0)$ & \\
\hline$\geq 20000$ & $64(62.7)$ & $112(56 \cdot 0)$ & \\
\hline Co-morbidity & $85(83.3)$ & $0(0)$ & $<0.001$ \\
\hline
\end{tabular}

Values in parentheses are percentages unless indicated otherwise; *values are mean(s.d.) (range). BMI, body mass index; NT, new Taiwan dollar.

$\dagger \chi^{2}$ test unless indicated otherwise; $\ddagger$ Student’s $t$ test. between the participants and non-participants, including BMI (mean(s.d.) $42 \cdot 3(7 \cdot 0)$ versus $41 \cdot 1(7 \cdot 7) \mathrm{kg} / \mathrm{m}^{2}$ ), age (mean(s.d.) $32 \cdot 1(9 \cdot 6)$ versus $31 \cdot 1(7 \cdot 7)$ years), sex $(62.7$ versus 67.2 per cent women), marital status (32.4 versus 34.5 per cent married), education (61.8 versus 51.7 per cent educated for more than 12 years) and employment (46.1 versus 35.3 per cent employed).

A total of 200 healthy subjects were selected. The demographic and clinical characteristics of the 102 patients with morbid obesity and 200 healthy controls are summarized in Table 1. Obese subjects had a lower employment rate $(P=0.002)$ and a higher rate of comorbidities $(P<0 \cdot 001)$ than healthy controls.

Before bariatric surgery QOL scores in the physical, psychological and social domains among patients with morbid obesity were significantly lower than those of the age-, sex-, education- and municipality-matched subjects (Table 2). QOL scores in the physical and psychological domains increased rapidly at 1 month after operation but decreased slightly from 3 to 6 months after surgery. Both of these scores gradually improved from 6 to 12 months after operation (Table 2, Fig. 1). The scores in the social domain showed a marked increase during the first month, remaining steady up to 6 months followed by a gradual increase. There was no significant change in QOL scores in the environment domain (Fig. 1). Several individual aspects that contribute to QOL changed markedly during the year (Fig. 2).

The mixed-effect model demonstrated that QOL scores in the physical, psychological and social domains improved significantly after surgery, associated with a falling BMI (Table 3). Many items in the physical domain, including 'pain and/or discomfort', 'mobility', 'activities of daily living' and 'dependence on medication or treatments', improved in parallel with the reduction in BMI. The physical domain was affected by the occurrence of

Table 2 Comparison of body mass index values and health-related quality of life scores from four domains between patients with morbid obesity before and after bariatric surgery, and age-, sex-, municipality-, marriage- and education-matched healthy control subjects

\begin{tabular}{|c|c|c|c|c|c|c|c|}
\hline & \multicolumn{5}{|c|}{ Patients $(n=102)$} & \multicolumn{2}{|c|}{ Healthy subjects $(n=200)$} \\
\hline & \multicolumn{5}{|c|}{ Time after operation (months) } & \multirow{2}{*}{$\begin{array}{c}\mathrm{BMI} \geq 25 \text { to }<32 \mathrm{~kg} / \mathrm{m}^{2} \\
(n=98)\end{array}$} & \multirow{2}{*}{$\begin{array}{c}\mathrm{BMI}<25 \mathrm{~kg} / \mathrm{m}^{2} \\
(n=102)\end{array}$} \\
\hline & 0 & 1 & 3 & 6 & 12 & & \\
\hline $\mathrm{BMI}\left(\mathrm{kg} / \mathrm{m}^{2}\right)$ & $42 \cdot 3(7 \cdot 0)$ & $38 \cdot 5(6 \cdot 8)$ & $35 \cdot 0(6 \cdot 1)$ & $31 \cdot 2(4.9)$ & $28 \cdot 4(6 \cdot 0)$ & $26 \cdot 9(2 \cdot 0)^{\star}$ & $20 \cdot 9(2 \cdot 1) \ddagger$ \\
\hline \multicolumn{8}{|l|}{ QOL score } \\
\hline Physical & $13 \cdot 5(2 \cdot 2)$ & $14 \cdot 5(2 \cdot 3)$ & $14 \cdot 5(2 \cdot 2)$ & $14 \cdot 3(2 \cdot 3)$ & $14 \cdot 8(2 \cdot 0)$ & $15 \cdot 4(2 \cdot 0)$ & $15 \cdot 6(1 \cdot 6)^{\star}$ \\
\hline Psychological & $11.6(2.9)$ & $12 \cdot 9(2 \cdot 7)$ & $13 \cdot 2(2 \cdot 7)$ & $12 \cdot 8(3 \cdot 1)$ & $13 \cdot 6(2 \cdot 7)$ & $13 \cdot 6(2 \cdot 3)$ & $14.0(1.9)$ \\
\hline Social & $13 \cdot 3(2 \cdot 5)$ & $14 \cdot 1(2 \cdot 3)$ & $14 \cdot 2(2 \cdot 4)$ & $14 \cdot 2(2 \cdot 5)$ & $14 \cdot 7(2 \cdot 5)$ & $13 \cdot 9(2 \cdot 1) \dagger$ & $14 \cdot 7(2 \cdot 1)$ \\
\hline Environment & $13 \cdot 5(2 \cdot 0)$ & $13.6(1.9)$ & $13 \cdot 8(2 \cdot 1)$ & $13 \cdot 8(2 \cdot 1)$ & $14 \cdot 2(2 \cdot 0)$ & $13 \cdot 2(2 \cdot 1) \ddagger$ & $13.9(2.0)$ \\
\hline
\end{tabular}

Values are mean(s.d.). BMI, body mass index; QOL, quality of life. ${ }^{*} P=0.022, \dagger P=0.025, \ddagger P<0.001$ versus results at 12 months after operation (Student's $t$ test). 


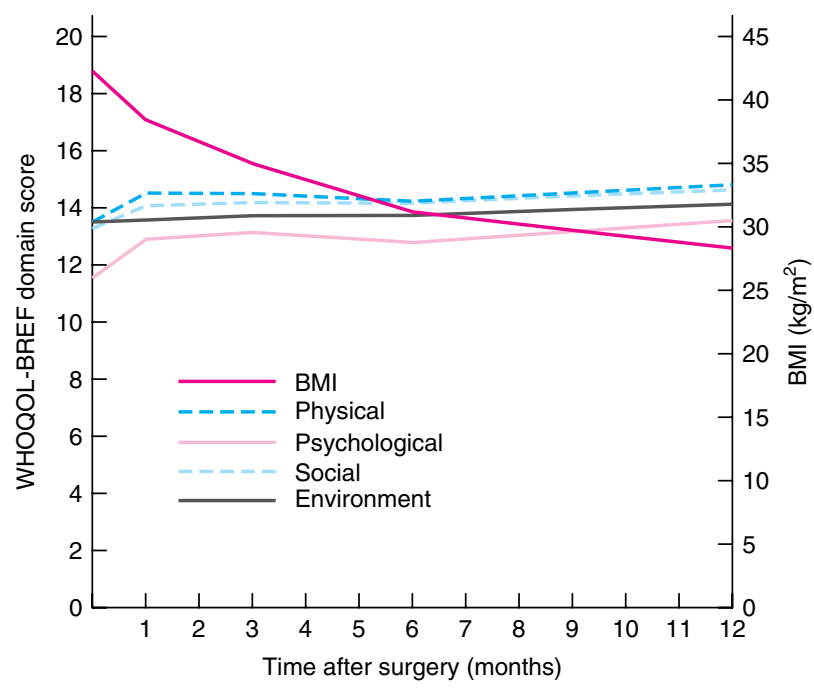

Fig. 1 Scores for physical, psychological, social and environment domains in World Health Organization Quality of Life - Brief (WHOQOL-BREF) and body mass index (BMI) changes after bariatric surgery

operation-related complications. QOL scores in the psychological domain were reduced by the presence of morbid obesity-related co-morbidities but increased by the reduction in BMI, employment and male sex. 'Positive feelings', 'self-esteem' and 'body image and appearance' were the specific aspects changed by bariatric surgery through a reduction in BMI. QOL scores in the social

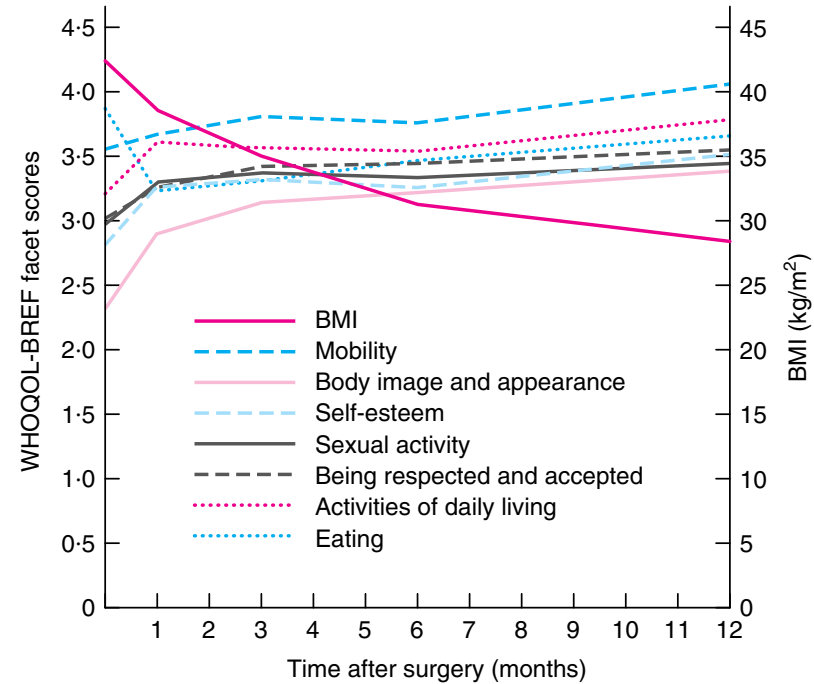

Fig. 2 Scores for specific aspects of quality of life in World Health Organization Quality of Life - Brief (WHOQOL-BREF) and body mass index (BMI) changes after bariatric surgery

domain were reduced by the occurrence of co-morbidities, but increased by loss of bodyweight and employment. Two items in the social domain, 'sexual activity' and 'being respected and accepted', were improved by weight reduction and employment (Table 3).

At 1 year, the mean BMI improved from 42.3 to $28.4 \mathrm{~kg} / \mathrm{m}^{2}$ and the mean loss of excess BMI was $80 \cdot 3$ per cent. Hypertension, GORD and abnormal liver

Table 3 Regression coefficients based on mixed-effect model analysis of each domain and item of health-related quality of life in patients with morbid obesity before and after bariatric surgery

\begin{tabular}{|c|c|c|c|c|c|}
\hline & $\begin{array}{c}\mathrm{BMI} \\
\left(\mathrm{kg} / \mathrm{m}^{2}\right)\end{array}$ & $\begin{array}{l}\text { Co-morbidity } \\
\text { (yes/no) }\end{array}$ & $\begin{array}{c}\text { Complication } \\
\text { (yes/no) }\end{array}$ & $\begin{array}{l}\text { Sex } \\
\text { (M) }\end{array}$ & $\begin{array}{l}\text { Employment } \\
\text { (yes } / \mathrm{no})\end{array}$ \\
\hline Physical & $-0.067(0.018) \ddagger$ & & $-1.158(0.352) \dagger$ & & \\
\hline Pain and/or discomfort & $-0.031(0.010) \dagger$ & & $-0.656(0.224) \dagger$ & & \\
\hline Mobility & $-0.023(0.007) \dagger$ & $-0.210(0.092)^{\star}$ & & & \\
\hline Activities of daily living & $-0.013(0.007)^{\star}$ & & $-0.428(0.146) \dagger$ & & \\
\hline Dependence on medication or treatments & $-0.019(0.008)^{\star}$ & & & & \\
\hline Psychological & $-0.046(0.021)^{\star}$ & $-0.829(0.270) \dagger$ & & $1.071(0.485)^{\star}$ & $1.280(0.524)^{*}$ \\
\hline Positive feelings & $-0.013(0.007)^{\star}$ & & & & \\
\hline Self-esteem & $-0.017(0.008)^{*}$ & $-0.263(0.099) \dagger$ & & $0.331(0.152)^{\star}$ & \\
\hline Body image and appearance & $-0.026(0.008) \dagger$ & $-0.385(0.102) \ddagger$ & & $0.342(0.145)^{\star}$ & \\
\hline Negative feelings & & $-0.224(0.097)^{\star}$ & & & $0.387(0.164)^{*}$ \\
\hline Social & $-0.039(0.019)^{*}$ & $-0.586(0.244)^{\star}$ & & & $1.077(0.436)^{*}$ \\
\hline Personal relationships & & $-0.191(0.087)^{\star}$ & & $0.314(0.142)^{\star}$ & \\
\hline Sexual activity & $-0.016(0.007)^{\star}$ & & & & $0.364(0.126) \dagger$ \\
\hline Being respected and accepted & $-0.018(0.007) \dagger$ & & & & $0.263(0.136)^{*}$ \\
\hline \multicolumn{6}{|l|}{ Environment } \\
\hline Financial resources & $-0.018(0.008)^{\star}$ & & & $0.410(0.157)^{\star}$ & \\
\hline Physical safety and security & & $-0.307(0.103) \dagger$ & & $0.398(0.145) \dagger$ & \\
\hline Eating what one likes & & & $-0.395(0.184)^{*}$ & & \\
\hline
\end{tabular}

Values in parentheses are standard errors. BMI, body mass index. ${ }^{*} P<0.050, \dagger P 0.010, \ddagger P<0.001$ (mixed-effect model analysis). 
Table 4 Effect of bariatric surgery on obesity-associated major co-morbidities

\begin{tabular}{|lcrr|} 
& & 12 months \\
& Preop. & postop. & $P^{*}$ \\
\hline Diabetes mellitus & $25(24.5)$ & $15(14.7)$ & 0.078 \\
Hypertension & $57(55.9)$ & $20(19.6)$ & $<0.001$ \\
Gastro-oesophageal reflux disease & $42(41.2)$ & $18(17.6)$ & $<0.001$ \\
Abnormal liver function & $28(27.5)$ & $5(4.9)$ & $<0.001$ \\
\hline
\end{tabular}

Values in parentheses are percentages. ${ }^{*} \chi^{2}$ test.

function improved significantly after bariatric surgery; although there was a fall in the number of diabetic patients, this did not reach significance (Table 4). Operationrelated complications, including gastrojejunal anastomotic stricture (12 patients), gastrojejunal anastomotic ulcer (9), upper gastrointestinal bleeding (1) and GORD (2), were treated endoscopically or with appropriate medication.

\section{Discussion}

Although bariatric surgery has been shown to reduce bodyweight, and improve QOL and co-morbidities $6,8,15,30,31$, this study is the first to show the detailed impact on QOL changes in the year after surgery. A mixed-effect model analysis was used to minimize confounding. Improvements were seen in various domains and aspects of QOL for the first 3 months, after which there was a slight downward trend in physical and psychological domains between 3 and 6 months that seemed to be associated with complications, followed by further improvement up to the end of the first year. The present findings corroborate other results ${ }^{30}$, showing that laparoscopic gastric bypass could improve both physical and mental health dimensions of the Short Form $36\left(\mathrm{SF}-36^{\circledR}\right.$; Medical Outcomes Trust, Waltham, Massachusetts, USA).

Once complications had been treated, QOL scores increased again from 6 to 12 months. The curve of dynamic changes in QOL scores in the psychological domain was similar to that in the physical domain. This might relate to 'self-esteem', for which scores were lowest 6 months after surgery. Scores in the social domain increased rapidly during the first month, and remained steady between 1 and 6 months after surgery. These corresponded to the sum of increased individual scores for 'being respected and accepted' and decreased scores for 'sexual activity'.

This analysis provides more detailed information to assist bariatric surgeons in controlling potential problems for patients during follow-up. For example, scores for 'pain and/or discomfort', 'self-esteem' and 'sexual activity' improve rapidly during the first 3 months after surgery, but decrease with the appearance of complications, gradually improving again when these complications are alleviated. Surgeons might therefore look for specific procedurerelated complications that tend to be troublesome between 3 and 6 months. Prompt treatment might mitigate some of the deterioration in QOL at this time. Patients can also be reassured that these aspects of QOL will improve.

The comparison between the average QOL of morbidly obese subjects and the healthy national population was informative. Although the mean physical domain score of the morbidly obese at 1 year after surgery was similar to that of control subjects whose BMI values were between 25 and $32 \mathrm{~kg} / \mathrm{m}^{2}$, it was still lower than the score for those with a BMI less than $25 \mathrm{~kg} / \mathrm{m}^{2}$. Mean scores in both psychological and social domains of the morbidly obese reached the same level as in healthy subjects whose BMI values were less than $25 \mathrm{~kg} / \mathrm{m}^{2}$.

This study has several limitations. Although the mixedeffect model controlled for most potential confounding factors associated with QOL, this study was not a randomized controlled trial so the magnitude of the possible placebo effect of surgery cannot be determined. The participation rate of 46.8 per cent raises concern about whether the sample was representative. As there were no significant differences between the participants and nonparticipants for most demographic factors and severity of obesity as measured by BMI, the likelihood of bias was probably small. Physical scores remained lower than those of control subjects with a BMI of less than $25 \mathrm{~kg} / \mathrm{m}^{2}$, and longer follow-up is needed before making inferences about bariatric surgery and long-term effects on QOL.

\section{Acknowledgements}

The authors thank Professor Jing-Shiang Hwang for statistical support. The authors declare no conflict of interest.

\section{References}

1 Ogden CL, Carroll MD, Curtin LR, McDowell MA, Tabak CJ, Flegal KM. Prevalence of overweight and obesity in the United States, 1999-2004. 7AMA 2006; 295 : $1549-1555$.

2 Sturm R. Increases in clinically severe obesity in the United States, 1986-2000. Arch Intern Med 2003; 163: 2146-2148.

3 Prentice AM. The emerging epidemic of obesity in developing countries. Int 7 Epidemiol 2006; 35: 93-99.

4 Lee WJ, Wang W. Bariatric surgery: Asia-Pacific perspective. Obes Surg 2005; 15: 751-757.

5 Huang KC. Obesity and its related diseases in Taiwan. Obes $\operatorname{Rev} 2008 ; 9$ : 32-34. 
6 Elder KA, Wolfe BM. Bariatric surgery: a review of procedures and outcomes. Gastroenterology 2007; 132: 2253-2271.

$7 \mathrm{Li}$ Z, Bowerman S, Heber D. Health ramifications of the obesity epidemic. Surg Clin North Am 2005; 85: 681-701.

8 Buchwald H, Avidor Y, Braunwald E, Jensen MD, Pories W, Fahrbach $\mathrm{K}$ et al. Bariatric surgery: a systematic review and meta-analysis. FAMA 2004; 292: 1724-1737.

9 Livingston EH, Fink AS. Quality of life: cost and future of bariatric surgery. Arch Surg 2003; 138: 383-388.

10 Chang CY, Hung CK, Chang YY, Tai CM, Lin JT, Wang JD. Health-related quality of life in adult patients with morbid obesity coming for bariatric surgery. Obes Surg 2008; [Epub ahead of print 8 May 2008].

11 US Department of Health and Human Services FDA Center for Drug Evaluation and Research; US Department of Health and Human Services FDA Center for Biologics Evaluation and Research; US Department of Health and Human Services FDA Center for Devices and Radiological Health. Guidance for industry: patient-reported outcome measures: use in medical product development to support labeling claims: draft guidance. Health Qual Life Outcomes 2006; 4: 79.

12 Delinsky SS, Latner JD, Wilson GT. Binge eating and weight loss in a self-help behavior modification program. Obesity (Silver Spring) 2006; 14: 1244-1249.

13 O'Brien PE, Dixon JB, Laurie C, Skinner S, Proietto J, $\mathrm{McNeil} \mathrm{J}$ et al. Treatment of mild to moderate obesity with laparoscopic adjustable gastric banding or an intensive medical program: a randomized trial. Ann Intern Med 2006; 144: 625-633.

14 NIH conference. Gastrointestinal surgery for severe obesity. Consensus Development Conference Panel. Ann Intern Med 1991; 115: 956-961.

15 Maggard MA, Shugarman LR, Suttorp M, Maglione M, Sugerman HJ, Livingston EH et al. Meta-analysis: surgical treatment of obesity. Ann Intern Med 2005; 142: 547-559.

16 Davis MM, Slish K, Chao C, Cabana MD. National trends in bariatric surgery, 1996-2002. Arch Surg 2006; 141: 71-74.

17 Steinbrook R. Surgery for severe obesity. N Engl 7 Med 2004; 350: $1075-1079$.

18 Glinski J, Wetzler S, Goodman E. The psychology of gastric bypass surgery. Obes Surg 2001; 11: 581-588.

19 Mathus-Vliegen EM, de Weerd S, de Wit LT.

Health-related quality-of-life in patients with morbid obesity after gastric banding for surgically induced weight loss. Surgery 2004; 135: 489-497.

20 Anonymous. Study protocol for the World Health Organization project to develop a Quality of Life assessment instrument (WHOQOL). Qual Life Res 1993; 2: 153-15921.

21 Anonymous. The World Health Organization Quality of Life Assessment (WHOQOL): development and general psychometric properties. Soc Sci Med 1998; 46: 1569-1585.

22 Anderson RT, Aaronson NK, Bullinger M, McBee WL. A review of the progress towards developing health-related quality-of-life instruments for international clinical studies and outcomes research. Pharmacoeconomics 1996; 10: $336-355$.

23 Anonymous. Development of the World Health Organization WHOQOL-BREF quality of life assessment. The WHOQOL Group. Psychol Med 1998; 28: 551-558.

24 Liou HH, Chen RC, Chen CC, Chiu MJ, Chang YY, Wang JD. Health related quality of life in adult patients with epilepsy compared with a general reference population in Taiwan. Epilepsy Res 2005; 64: 151-159.

25 Yang MH, Chen YM, Kuo BI, Wang KY. Quality of life and related factors for people living with HIV/AIDS in Northern Taiwan. 7 Nurs Res 2003; 11: 217-226.

26 Hsu C, Wang JD, Hwang JS, Tien HF, Chang SM, Cheng AL et al. Survival-weighted health profile for long-term survivors of acute myelogenous leukemia. Qual Life Res 2003; 12: 503-517.

27 Hsiung PC, Fang CT, Chang YY, Chen MY, Wang JD. Comparison of WHOQOL-BREF and SF-36 in patients with HIV infection. Qual Life Res 2005; 14: 141-150.

28 Yao G, Chung CW, Yu CF, Wang JD. Development and verification of validity and reliability of the WHOQOLBREF Taiwan version. 7 Formos Med Assoc 2002; 101: $342-351$.

29 Lin SH. Field collection and completeness of data in the National Health Interview Survey. NHIS Brief Communication 2002; 4: 1-8.

30 Nguyen NT, Goldman C, Rosenquist CJ, Arango A, Cole CJ, Lee SJ et al. Laparoscopic versus open gastric bypass: a randomized study of outcomes, quality of life, and costs. Ann Surg 2001; 234: 279-289.

31 Suter M, Paroz A, Calmes JM, Giusti V. European experience with laparoscopic Roux-en- $Y$ gastric bypass in 466 obese patients. Br F Surg 2006; 93: 726-732. 